death and lower the severity of injuries sustained in road traffic accidents.

Objectives The main objective of our study was to explore the knowledge, attitude and practice of parents of newborn babies regarding infant or child car restraints during their homeward trip from the hospital. The secondary aim was to evaluate the reasons behind non-compliance to infant and child car restraints amongst those who intended to travel home in a motor vehicle, and compare our findings with a systematic review of child car seat restraints.

Methods A 22-item self-administered questionnaire was completed by a convenience sampling of 200 parents of babies about to be discharged from a well-baby nursery in Singapore during the 4-month study period. Parents of babies who were admitted to the Neonatal Intensive Care Unit, transferred to other hospitals or passed on, along with unwell mothers, were excluded.

Results Out of 200 parents, 98.5\% intended to bring their newborn baby home by a motor vehicle. Parental knowledge of the local legislation on child car restraints was generally high (74\%), with the majority aware of the correct location (93.5\%) and orientation inside the vehicle (72\%). Although 106 parents were already in possession of a child car restraint, only $81(76 \%)$ intended to use it for the homeward journey. The remaining chose not to because of a personal belief, misperception of risk and/or logistical difficulty. Ownership and usage of child car restraints was positively correlated with household income.

Conclusions The surveyed parents generally had a high level of knowledge regarding child car restraints. However, the misperception of need which led to inconsistent practice was worrying. A multi-faceted approach, including parental education and regulatory requirements, are important to increase compliance to child and infant car seat restraints in Singapore.

\section{KNOWLEDGE, ATTITUDES AND PRACTICES TOWARDS BREASTFEEDING AMONGST PAEDIATRIC HEALTHCARE PROFESSIONALS}

Mercy Murinye Magwenzi. UK

\subsection{6/bmjpo-2021-RCPCH.131}

Background The World Health Organisation recommends exclusive breastfeeding of infants for six months when complementary feeds are introduced whilst continuing breastfeeding for up to two years of age and beyond. Ongoing predictable support to breastfeeding mothers is essential to improve breastfeeding rates and paediatric hospital doctors and nurses have an opportunity to provide this support when breastfed infants are admitted to their wards.

Objectives This study aimed to assess the breastfeeding knowledge, attitudes, and practices amongst paediatric healthcare professionals in a large teaching hospital in the UK.

Methods We conducted a qualitative study through a selfadministered online questionnaire. Participants were doctors, nurses, nursery nurses and student nurses working in the paediatric department at the time of the study. There were 32 respondents, 14 of these were doctors.

Results

- Emergent themes were identified and analysed across all interviews. Five themes emerged: Breastfeeding knowledge and training, the influence of paediatric work experience, practitioner attitude towards breastfeeding, role in breastfeeding and practice in supporting breastfeeding.

- Almost all the participants had good levels of breastfeeding knowledge though only 50\% of doctors had received formal breastfeeding training and were relying on personal experience whilst $70 \%$ of nurses had received training.

- All participants had a positive attitude towards breastfeeding with nurses mostly indicating comfort in the role of supporting mothers. A significant proportion of doctors indicated a lack of conviction in their ability to support breastfeeding mothers.

- Inconsistent advice would be offered to mothers about bottle feeding by different practitioners.

Conclusions 7 out of 14 doctors had no breastfeeding training and doctors were found to be less confident in the role of supporting breastfeeding mothers. There was good knowledge base amongst all the healthcare professionals however the actual practice of advice offered in supporting mothers was inconsistent amongst practitioners.

We hope that the results of this study will encourage formal breastfeeding education to be offered for all health care professionals working in paediatrics and the development of departmental breastfeeding policies to enable consistency whilst improving the care offered to patients and their families.

\section{TRIAL PROFILE: COMMUNITY ENABLED READINESS FOR FIRST 1000-DAYS LEARNING ECOSYSTEM (CRADLE)}

Joyce Teo, See Ling Loy, Sing Zhi Kee, Thilagamangai, Oh Moh Chay, Kee Chong Ng. Singapore

\subsection{6/bmjpo-2021-RCPCH.132}

Background The first 1000 days presents a unique opportunity to enhance a child's ability to grow, learn and thrive which will have a long term impact in a child's future.

Objectives The Community enabled ReAdiness for first 1000Days Learning Ecosystem (CRADLE) is a trial designed to develop a self-learning eco-community from pregnancy to early-childhood to promote parenting self-efficacy (PSE) and improve health outcomes for first-time families. Here, we describe the trial protocol and provide updates thus far.

Methods CRADLE is a parallel, three-arm randomised controlled trial which targets to recruit 750 pregnant women from KK Women's and Children's Hospital (KKH), Singapore. Participants are randomly assigned to receive: (1) standard routine care; (2) behavioural nudges and engagement via a social media platform; or (3) midwife-led continuity care involving direct individual engagement with midwives throughout pregnancy, to 6-month post-delivery.

We hypothesize that the use of virtual targeted nudging tool in the form of text messages along with social media (arm 2), or face-to-face care from midwives supplemented with individualised teleconferencing sessions ( $\operatorname{arm} 3$ ) in the first 1000 days will lead to a better maternal PSE and health outcomes in both mother-child, compared with those who are receiving standard routine care (arm 1).

The primary outcome is PSE, while the secondary outcomes include birth experience, mental wellness, feeding practice and nutritional status of mother-child. Participants are followed-up 